Published in final edited form as:

ACS Chem Biol. 2020 February 21; 15(2): 575-586. doi:10.1021/acschembio.0c00019.

\title{
Integrative $x$-ray structure and molecular modeling for the rationalization of procaspase-8 inhibitor potency and selectivity
}

\author{
Janice H. Xu ${ }^{1,2}$, Jerome Eberhardt ${ }^{2}$, Brianna Hill-Payne ${ }^{4}$, Gonzalo E. González-Páez ${ }^{1,2}$, \\ José Omar Castellón ${ }^{4}$, Benjamin F. Cravatt ${ }^{3}$, Stefano Forli ${ }^{2,}{ }^{*}$, Dennis W. Wolan ${ }^{1,2,{ }^{*}, \text { Keriann }}$ \\ M. Backus ${ }^{4,}$ \\ ${ }^{1}$ Department of Molecular Medicine, The Scripps Research Institute, 10550 North Torrey Pines \\ Road, La Jolla, CA 92037 \\ 2Department of Structural and Computational Biology, The Scripps Research Institute, 10550 \\ North Torrey Pines Road, La Jolla, CA 92037 \\ ${ }^{3}$ Department of Chemistry, The Scripps Research Institute, 10550 North Torrey Pines Road, La \\ Jolla, CA 92037 \\ ${ }^{4}$ Departments of Biological Chemistry and Chemistry and Biochemistry, David Geffen School of \\ Medicine, University of California, Los Angeles, 405 Hilgard Avenue, Los Angeles, CA 90095
}

\begin{abstract}
Caspases are a critical class of proteases involved in regulating programmed cell death and other biological processes. Selective inhibitors of individual caspases, however, are lacking, due in large part to the high structural similarity found in the active sites of these enzymes. We recently discovered a small-molecule inhibitor, 63-R, that covalently binds the zymogen, or inactive precursor (pro-form), of caspase-8, but not other caspases, pointing to an untapped potential of procaspases as targets for chemical probes. Realizing this goal would benefit from a structural understanding of how small molecules bind to and inhibit caspase zymogens. There have, however, been very few reported procaspase structures. Here, we employ x-ray crystallography to elucidate a procaspase- 8 crystal structure in complex with $63-\mathrm{R}$, which reveals large conformational changes in active-site loops that accommodate the intramolecular cleavage events required for protease activation. Combining these structural insights with molecular modeling and mutagenesis-based biochemical assays, we elucidate key interactions required for 63-R inhibition of procaspase-8. Our findings inform the mechanism of caspase activation and its disruption by small molecules, and, more generally, have implications for the development of small molecule
\end{abstract}

“Corresponding Authors: forli@scripps.edu, wolan@scripps.edu, and kbackus@mednet.ucla.edu. Author Contributions

KMB, DWW, SF, and BFC conceived of the project. JHX and GEGP solved the $\mathrm{x}$-ray structure. SF and JE conducted computational studies. KMB, BHP and JOC conducted mutagenesis, gel-based ABPP, and CD experiments. KMB, SF, and DW designed experiments and analyzed data. KMB, SF, and DW wrote the manuscript with assistance from JHX and JE.

The authors declare no competing financial interests.

ASSOCIATED CONTENT

Supporting Information

The Supporting Information is available free of charge on the ACS Publications website at DOI: \#\#\#\#\#\#

Detailed information about synthetic procedures and x-ray crystallography statistics (PDF) 
inhibitors and/or activators that target alternative (e.g., inactive precursor) protein states to ultimately expand the druggable proteome.

\section{Graphical Abstract}

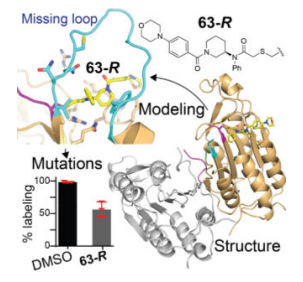

\section{Keywords}

Procaspase-8; crystallography; modeling; mutational analysis; covalent inhibitor

Caspases are cysteine proteases responsible for driving many cellular activities and are most well-known for inducing and executing apoptosis. However, caspases are also involved in promoting cellular activation, differentiation, and inflammation events, and their role in these processes is less understood. ${ }^{1-6}$ Aberrant proteolysis by this family of proteases can have devastating results, including the proliferation of cancers, ${ }^{7}$ neurodegeneration, ${ }^{8,9}$ and immunological disorders. ${ }^{10,11}$ As such, caspases are heavily regulated within the cellular environment. They are synthesized as a single polypeptide chain with an unformed active site and are maintained in this inactive state until a cellular stimulus triggers proteolysis of the scissile bond after distinct aspartate residues. ${ }^{12,13}$

Efforts to develop selective caspase inhibitors and molecular probes have largely focused on compounds that target the active caspase conformers. ${ }^{14}$ Unfortunately, as numerous molecular structures of active caspases have revealed, this family of proteases share highly conserved active site and molecular architecture, leading to significant overlap in substrate specificity. ${ }^{14,15}$ This general conservation is supported by in vitro studies using short fluorogenic peptide-based substrates and inhibitors with electrophilic warheads. ${ }^{16,17}$ Therefore, peptide-based inhibitors, such as the commonly used zVAD-fluoromethyl ketone (zVAD-fmk), are hampered by limited selectivity profiles against both caspase- and noncaspase proteases. Given the rapid rate of activation of most caspases and the subsequent cleavage of downstream executioner caspases, inhibition of active conformers will likely fail to fully block the ensuing consequences of caspase activation. Allosteric inhibitors, such as compounds that target the caspase dimer interfaces have been proposed as an alternative strategy to improve the selectivity profile of caspase inhibitors. ${ }^{18-20}$ To date, allosteric caspase inhibitors are only available for caspases- $1,-6$, and -7 .

The promiscuity and incomplete inhibition of active caspase inhibitors could be circumvented by an alternative strategy of targeting procaspases. The maturation of the pro(inactive or zymogen) enzymes is the primary mechanism of caspase regulation in the cellular environment (Figure 1A). Although the specific molecular mechanism of activation for individual caspases remains somewhat unresolved, studies have established that, for 
initiator caspases (i.e., caspases- $2,-8,-9$, and -10 ), proteolysis is triggered by transient proximity-induced homodimerization followed by intramolecular proteolysis. ${ }^{21,22}$ Executioner caspases (i.e., caspases-3 and -7) are subsequently subjected to proteolysis by activated initiator caspases. Of the 12 known human caspases, only procaspases- $1,-3,-6$, and -7 have $\mathrm{x}$-ray crystal structures. ${ }^{23-26}$ An NMR structure of the procaspase- 8 monomer has also been reported. ${ }^{27}$ Consequently, our understanding of the molecular mechanisms of caspase activation, particularly, the determination of whether the processing of caspases occurs in cis (intramolecular) or in trans (intermolecular) have been limited. Studies have also indicated that the somewhat cryptic enzymatic activity of the unprocessed procaspase likely contributes to a variety of non-apoptotic activities assigned to caspases. ${ }^{27-29}$

We recently identified several non-peptidic, selective inhibitors of procaspase- $8,{ }^{30}$ an initiator caspase that contributes to both extrinsic (Fas ligand-induced) and intrinsic apoptosis. ${ }^{31,32}$ These compounds (Figure 1B) function by irreversible alkylation of the catalytic cysteine in the inactive precursor state of the enzyme, thus blocking activation and subsequent cleavage of downstream executioner caspases-3. The most potent compound, 63$\boldsymbol{R}$, featured an alpha-chloroacetamide electrophile coupled to an $N, N$-disubstituted $(R)-3$ aminopiperidine phenyl core that is further functionalized with a 4-morpholinobenzoyl substituent. Targeting the procaspase conformer is akin to drugging inactive conformations of kinases, a method that has yielded potent and selective inhibitors of several kinases, including the Abl kinase inhibitor imatinib. ${ }^{33-36}$

We report the crystal structure of procaspase- 8 in complex with $\mathbf{6 3 - R}$, which reveals that 63$\boldsymbol{R}$ binds in a pose distinct from that characterized for inhibitors of processed, active forms of caspases. The structure also uncovers large conformational changes in active-site loops that accommodate the intramolecular cleavage events required for caspase-8 processing and activation. To identify and validate key residues involved in ligand recognition and binding, including those not resolved in the crystal structure, we combined molecular modeling with point mutagenesis and binding studies. This hybrid computational-biochemical approach uncovered residues involved in recognition of $\mathbf{6 3 - R}$, another less potent inhibitor $\mathbf{7}$, and an alkyne-containing clickable analog of 7 (61). Our findings also aided in the rationalization of an inactive, structurally related compound (62) (Figure 1B). We anticipate that the integrated and interdisciplinary strategy described herein will find widespread utility for the identification and functional validation of key structural features missing from crystal structures.

\section{Results and Discussion}

\section{X-ray structure of procaspase-8 compared to active caspase-8.}

We determined the x-ray crystal structure of procaspase-8 (residues 223-479) in complex with 63-R to $2.88 \AA$ resolution (PDB 6PX9) (Figure 2 and Table S1). The final $\mathrm{R}_{\text {cryst }}$ and $\mathrm{R}_{\text {free }}$ values were $28.9 \%$ and $36.6 \%$, respectively, with $89 \%$ of the residues residing the most favored region of the Ramachadran plot (Table S1). The structure solution contains 6 molecules per asymmetric unit that form 3 biologically relevant homodimers. Residues 362$388,409-419$, and 453-460 of all 6 subunits lacked interpretable density. All three missing 
sequences are localized to loops that are exposed to solvent channels, and the missing density suggests these loops are highly flexible.

Superposition of procaspase- 8 with the structure of active caspase- 8 in complex with Ac-3Pal-D-BhLeu-hLeu-D-AOMK (PDB 4JJ7) shows the zymogen core scaffold is highly conserved with the active conformer (Figures $2 \mathrm{~A}$ and $\mathrm{B}$ ). The average main-chain rootmean-square deviation (RMSD) is $0.39 \AA$ (191/256 procaspase- $8 \mathrm{Ca}$ ). The average RMSD is $0.93 \AA$ for all atoms, with a maximum of $2.43 \AA$ (residues $223-358,397-403,421-452$, 463-478). The most significant conformational change with respect to the active form is loop 1 (residues 389-396). In the procaspase-8 structure, loop 1 is well positioned over the dimer interface, as demonstrate by clear electron density (Figures $2 \mathrm{C}$ and $\mathrm{D}$ ), and this orientation is similarly observed for loop 1 in the procaspase- 7 crystal structure and procaspase-8 NMR solution structure. ${ }^{23,27}$ Upon maturation, loop 1 flips approximately $180^{\circ}$ upon cleavage of the activation linker (residues 375-384) and results in the N-terminal region of loop 1 (residues 359-365) contributing key residues to the mature active site, while the C-terminal region of loop 1 (residues 390-396) is solvent exposed (Figures 2A and C). The primary interactions that position loop 1 are unique to each of the homodimer subunits and thus account for the dimer interface residing on a non-crystallographic symmetry axis. Interestingly, loop 1 of both subunits are positioned by residues within loops 1, 2, and 3 of the opposing subunit. The side chain of Arg391 and the main-chain carbonyl of Ile393 of subunit B are both within hydrogen bonding distance to the side chain of Gln 465 of subunit A (Figure 2E). Likewise, both the side chain and main-chain carbonyl of Asp395 from subunit B interact with the main-chain amide of Met463 from subunit A (Figure 2E). Conversely, the Asp395 side chain of subunit A forms potential hydrogen bonds with the side chain of Thr405 and main-chain amide from Gln462 of loops 2 and 3, respectively, from subunit B. An important consequence of the intact procaspase- 8 loop 1 is the $3.1 \AA$ shift (as measured by $\mathrm{Ca}$ ) of the catalytic Cys360 residue relative to the activated state (Figures 2F and S1). The displacement of Cys360 partially occludes the side chain from solvent exposure as well as misaligns the thiol with respect to His317, which is critical to promoting the nucleophilicity of Cys360 in the active caspase- 8 structure (Figure 2F).

In addition to the loop 1 rearrangement, loop 2 (missing residues 409-419) likely has a large conformational shift from the inactive to active conformers. In the active state, residues in loop 2 provide key active site pockets that are required for the recognition and positioning of the non-prime side region of peptide/protein substrates (C-terminal to the scissile bond) (Figure 2A). Despite much of loop 2 lacking electron density in the procaspase-8 structure (Figures $2 \mathrm{G}$ and S1), the N-terminal residues $404-408$ of loop 2 form a $\beta$-strand that provides a hydrogen-bond network with the neighboring strand containing Cys360 (Figures $2 \mathrm{~B}$ and $\mathrm{S} 1$ ). As such, the trajectory of the beginning residues within the loop suggest a distinct conformational orientation compared to the active state. Unfortunately, no residues that comprise loop 3 (residues 453-462) are resolvable in the procaspase structure, suggesting that this region is quite flexible. 


\section{Procaspase-8 interactions with 63- $R$.}

Crystallization of precursor forms of caspases has likely proven technically challenging due to their structural flexibility, and, in this regard, the procaspase- 8 structure appears to have been facilitated by covalent modification with $\mathbf{6 3 - R}$. Electron density was visible for

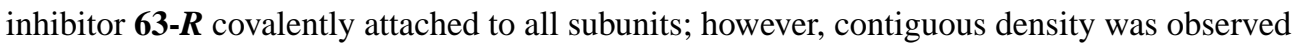
only in subunit B and we opted to model 63- $\boldsymbol{R}$ into this monomer only (Figures 3A and S2). We also subjected the procaspase- 8 crystals to LC-MS/MS analysis to verify that the protease was modified by 63- $\boldsymbol{R}$. Crystals were harvested, washed, solubilized in urea, and digested with trypsin. The trypsin-digested peptides were analyzed by liquid chromatography tandem mass spectrometry using an Q Exactive ${ }^{\mathrm{TM}}$ mass spectrometer. The

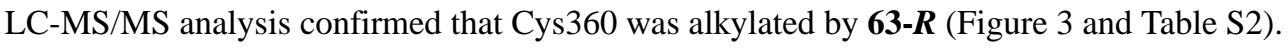

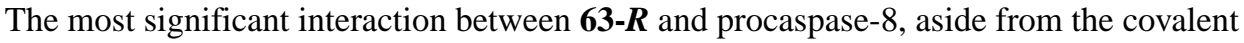
bond between the catalytic Cys360 and the inhibitor, is a weak hydrogen bond formed between the side chain of His264 and the oxygen on the morpholino group (Figure 3B). A further hydrogen bond is formed with the Asn261 with an adjacent subunit in the crystal lattice (Figure 3B). Due to the lack of any other polar interactions between the ligand and protein, we hypothesize that the driving force for the procaspase-8-ligand complex is primarily due to hydrophobic interactions. For example, the phenylamine is nestled within a pocket formed by Gln358, Arg260 and Trp420 (Figure 3B). The piperidine is positioned by the side chain of Trp420 and the benzoyl group has minimal interactions with the protein, leaving the carbonyl exposed to solvent. The morpholino group of $\mathbf{6 3 - R}$ is sandwiched into a pocket formed by residues His 264 and Leu265 (Figure 3B). This near complete absence of hydrogen bonding interactions is unexpected given the relative potency of the optimized lead inhibitor. Although it is possible that the compound is recognized purely based on hydrostatic interactions, another explanation is that the compound's pre-alkylation binding pose is distinct from the final pose observed in the $\mathrm{x}$-ray structure. We believe that the density of the ligand in subunit $\mathrm{B}$ is likely continuous due to the additional stability afforded by the potential crystal contact network. The ligand may also reduce the entropy of crystallization by stabilizing the crystal lattice. Superposition of active caspase- 8 and procaspase- 8 structures show that loop 2 residues $412-418$ would directly overlay with the

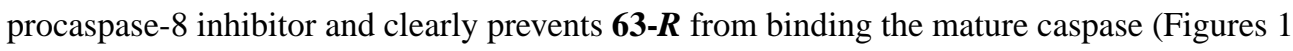
and $\mathrm{S} 1$ ).

\section{Procaspase-8 loop modeling.}

We next used homology modeling and docking to generate a complete model of the structure of procaspase- 8 bound to inhibitors $\mathbf{6 3 - R , 7}$, as well as the inactive compound $\mathbf{6 2}$. We generated approximately 5,000 models of procaspase- 8 to gain insight into the possible conformations of the missing active site loops 1 (residues 362-388), 2 (residues 409-419), and 3 (residues 453-460) and their respective interactions with 63- $R$. The modeling was restricted to the missing regions from the crystal structure and all residues with observable electron density were fixed in position. These models were used to predict $\mathbf{6 3 - R}$-binding residues that lacked electron density in the $\mathrm{x}$-ray structure. Out of the 5,000 models of the homodimer comprised of subunits A and B, we selected the best 1,000 models based on the 
energy score provided by MODELLER to explain and rationalize the inhibitor SAR and the respective selectivity of the molecules for procaspase- 8 .

Our analysis confirmed the identities of all residues in close contact ( $\leq 4 \AA$ ) with $\mathbf{6 3 - R}$ and suggested additional contacts from residues within the floppy regions of the loops, including most notably Asn408 (side-chain occupancy: 25.9\%; main-chain occupancy: 5.8\%); b) Cys409 (21.3\%; 6.4\%); and c) Asn407 (4.9\%; 45.5\%) from loop 2 (Figure 4). These residues are all in close contact with the phenylamide moiety of $\mathbf{6 3 - R}$ (Figure 4 ). In addition, we predict a number of main chains and side chains from loop 2 residues also transiently interact with 63- $\boldsymbol{R}$ with an occupancy $\leq 10 \%$, such as Pro415, Ser411, Ala416, Arg413 (ranked in decreasing order). We observed that the flexibility of loop 2 likely stems from Gly418, which does not interact directly with 63-R. Residues in loops 1 (with the exception of Cys360) and 3 are predicted to have no interactions with $\mathbf{6 3 - R}$ (Figure 4). It is important to highlight limitations of the models, which are based on the crystallographic template, and therefore only missing loops are considered as flexible, while the rest of the protein and the inhibitor are fixed in position. In non-crystallographic condition, the position of $\mathbf{6 3 - R}$ and the flanking loop 250-266 are expected to present additional flexibility and may limit the accuracy in the binding contributions by specific residues, and the resulting consequences of 63- $\boldsymbol{R}$ binding predicted to occur with mutational analysis. Our prediction accuracy is inversely proportional with respect to their distances from the crystallographically resolved residues. The residue occupancy calculated from the models was used to guide the selection and prioritization of side-chain mutagenesis to rationalize inhibitor SAR via perturbation of procaspase- 8 conformational stability/dynamics and/or introduction of steric clashes between 63- $\boldsymbol{R}$ and the procaspase- 8 binding site. Both methods of perturbance would result in a measurable and quantitative reduction in ligand binding.

\section{Point mutagenesis and compound binding studies.}

We next aimed to biochemically validate our structural model (Figure 4) and determine if the model accurately predicts residues critical for molecular recognition of electrophilic compounds that covalently label Cys360 in the specific active-site conformation formed in procaspase-8 (63- $\boldsymbol{R}$ and 7$)$. We also sought to rationalize the previously observed inactivity of compound $62 .{ }^{30}$ We theorized that residues proximal to $\mathbf{6 3 -} \boldsymbol{R}$ would be partially responsible for the potency and selectivity of compound binding and that mutation of these residues would alter compound affinity for procaspase-8. Among the residues within the procaspase- 8 active site, the following residues were prioritized for mutational analysis, including: a) Arg260 (predicted to increase the nucleophilicity of Cys360); b) Cys409, the backbone of Asn407, and to a lesser extent Arg258 (predicted to form the compound binding pocket lid); c) Asp266, Gln358 and Trp420 (comprise the bed of the binding site, but do not directly hydrogen bond with either 63-R and 7) - also notable, Asp266 and Gln358 also contribute to the hydrogen-bond network that likely stabilizes the Cys360 thiolate; d) His264 predicted to form a putative hydrogen bond with the morpholino group of the ligand; and Asn261, which was not predicted to interact with the ligand outside of the crystal lattice, and should then serve as a control mutation. All mutant proteins were generated on the uncleavable procaspase- 8 construct (see Experimental Section for details). As the Asn407 and Cys409 side chains were hypothesized to form a pocket, both residues 
were mutated to larger, bulkier groups (N407W and C409W, respectively) to eliminate the pocket and block compound binding. All other resides were mutated to alanine, including R260A, H264A, N261A, D266A, Q358A, and W420A. Unfortunately, the N407W and D266A constructs failed to yield soluble, folded proteins and were removed from further study.

Using a competitive gel-based activity-based protein profiling (ABPP) assay, we assessed the ability of the W420A and the H264A proteins to bind to compounds 7 and 63-R (Figure S3). Briefly, recombinant mutant proteins in cellular lysates were incubated with the indicated compounds at the indicated concentrations for $1 \mathrm{~h}$. The mixture was then chased with the alkyne-containing clickable analog of compound $7(61$ at $10 \mu \mathrm{M})$. Direct blockage of procaspase-8 labeling by 61 with pre-incubations in the presence of $\mathbf{6 3 - R}$ or $\mathbf{7}$ was visualized by $\mathrm{Cu}(\mathrm{I})$-catalyzed azide-alkyne cycloaddition (CuAAC or "click") conjugation to rhodamine-azide followed by SDS-PAGE and in-gel fluorescence analysis. We were surprised to observe no difference in compound labeling of the W420A compared to the wild-type procaspase-8 (Figure S3A). Next, we chose to investigate the contributions of His264, which can form a labile hydrogen bond with the ligand in the crystal structure. The H264A mutant protein showed comparable compound labeling to that observed for the wildtype procaspase (Figure S3B), confirming the negligible contribution of this interaction to ligand binding.

We hypothesized that the dynamics of the active site loops might contribute to compound binding in a manner that would not be captured by the $\mathrm{x}$-ray structure based on these unexpected results. Despite the lack of interactions between Gly418 and 63- $\boldsymbol{R}$ in our modeling studies, we still postulated that the mutation to an alanine would affect the loop dynamics and potentially alter the ligand binding. As such, we added the G418A and G418L mutants to our panel of proteins to test. Competitive gel-based ABPP experiments were performed at a single compound concentration of $10 \mu \mathrm{M}$, where $\mathbf{6 3 - R}$ and $\mathbf{7}$ completely ( $>90 \%)$ or partially ( $25 \%$ ) labeled procaspase-8, respectively, and $\mathbf{6 2}$ did not label, consistent with our previous study (Figure 5A). ${ }^{30}$ As shown previously, 63- $\boldsymbol{R}$ fully competes for labeling of procaspase- 8 by $\mathbf{6 1}$, indicating a high-potency labeling event at $10 \mu \mathrm{M} .7$ affords $\sim 75 \%$ decrease in labeling by $\mathbf{6 1}$, and $\mathbf{6 2}$ exhibits no appreciably competition (Figure 5B; procaspase-8 gel band). Quite surprisingly given its relative distance from the $\mathbf{6 3 - R}$ (about $9.7 \pm 1 \AA$ on average across the models), the G418A mutant exhibited striking changes in SAR across the compounds tested. G418A did not alter labeling by the 4-aminopiperidino containing 7 but did significantly decrease competition of 61 by the 3-aminopiperidino containing $\mathbf{6 3 - \boldsymbol { R }}$ (Figures 5A, B, and C). The relative sensitivity of the protein to mutations at the loop 2 hinge region is, perhaps, not surprising, given the significant repositioning of this loop to form the active conformation. In fact, the portion of loop 2 that forms part of the active site of active caspase- 8 and occupies the same space as the covalent inhibitor in the procaspase- 8 structure. This suggests that the inhibitor may be recognized by the protein as a mimic of this active site loop. While not conclusive, these results indicate that the dynamics and flexibility of loop 2 may contribute to the improved potency of $\mathbf{6 3 - R}$ with respect to 7 (prior calculated $\mathrm{IC}_{50 \mathrm{app}}$ of 0.7 and $5.0 \mu \mathrm{M}$, respectively. ${ }^{30}$ ) 
Using our competitive ABPP assay, we next assessed the ability of the procaspase-8 mutant panel (N261A, C409W, Q358A, R260A, and R258A) to bind compounds 7, 62, and 63-R (Figures 5A,D-I, S4, and S5). The R260A mutation, which is soluble and folded comparably to WT, as indicated by circular dichroism (CD), showed no appreciable labeling by 7 , consistent with R260 forming a key activating hydrogen bond with the catalytic Cys360 thiol. The bulky $\mathrm{C} 409 \mathrm{~W}$ mutation, which is predicted to partially occlude the binding site, significantly decreases labeling by $\mathbf{6 1}$ and nearly completely blocks competition by pretreatment with 7 or 63-R and agrees with the loop modeling. Mutation of Arg258, suspected to form part of the lid, did not significantly decrease the apparent potency of compound 7 or 63- $\boldsymbol{R}$. These results are consistent with the low occupancy of Arg258 around 63- $\boldsymbol{R}$ and lack of direct contacts with the ligand. The Q358A mutation, significantly decreased the intensity of protein labeling by 61 and modestly reduced competition of 61 by both $63-R$ and 7 , which is consistent with our model that Q358, together with other residues, forms the bed beneath the compound, but does not directly hydrogen bond to the ligand. While the N261 A mutation caused a slight decrease in $\mathbf{6 1}$ labeling, the mutation did not significantly alter the

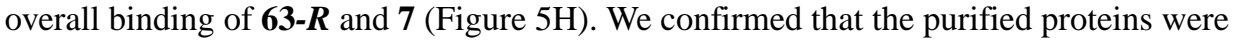
folded properly as measured by CD (Figure S4A). Similar protein concentrations for all mutant proteins in lysates were further confirmed by immunoblot (Figure S4B).

We next calculated the apparent $\mathrm{IC}_{50}$ values of labeling of the Q358A, C409W and G418A mutant proteins by $\mathbf{6 3 - R}$ and $\mathbf{7}$. As with the single dose experiments, proteins were spiked into lysates and labeled treated with either $63-R$ or 7 at a range of concentrations (500 nM $100 \mu \mathrm{M})$ followed by labeling with $\mathbf{6 1}(10 \mu \mathrm{M})$ and the apparent IC I0 $_{0}$ values (Figures 5I, S6, and S7) were calculated from the competition of labeling by $\mathbf{6 1}$. For the G418A mutation there is no significant change in the $\mathrm{IC}_{50}$ of 7 . In contrast, $\mathrm{G} 418 \mathrm{~A}$ affords a 10 -fold increase in the apparent $\mathrm{IC}_{50}$ of $\mathbf{6 3 - R}$ from $0.75 \mu \mathrm{M}$ for procaspase-8 (95\% confidence interval (CI), $0.62-0.94)$ to $7.14 \mu \mathrm{M}$ for the G418A mutant protein (95\% CI, 3.12-14.7), which decreases the potency of $\mathbf{6 3 - R}$ to approximate that of $\mathbf{7}$, which labels procaspase- 8 with an apparent $\mathrm{IC}_{50}$ value of $4.1 \mu \mathrm{M}(95 \% \mathrm{CI}, 2.94-5.80)$. C409W significantly increases the $\mathrm{IC}_{50}$ values of both 7 and 63-R, whereas Q358A only modestly alters the apparent $\mathrm{IC}_{50}$ values of both $\mathbf{6 3 - \boldsymbol { R }}$ and 7. All mutant proteins remained resistant to labeling by control probe $\mathbf{6 2}$, which showed no appreciable competition of labeling by $\mathbf{6 1}$, consistent with our previous studies. Finally, we investigated whether the four mutations that affected probe labeling of the pro-enzyme (Q358A, G418A, R260A, and C409W) would also impact the activity of the active protease. We found that all four residues impact the expression and activity of the recombinant protein, further supporting their functional importance (Figure S8).

\section{Pose prediction studies by molecular docking.}

To further verify and test the $\mathrm{x}$-ray structure and mutagenesis data, we performed silico

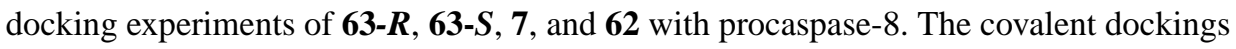
were based on the assumption that the four structurally related compounds should all assume similar covalently bound poses. As such, the position of the $\mathbf{6 3 - R}$ phenylamine was assigned as having the highest structural complementarity to the procaspase- 8 binding site and was used as a reference anchor for the docking analysis. The predicted position of the inactive $\mathbf{6 2}$ 1-naphthyl was used for comparison with the rest of the molecule assuming a similar 


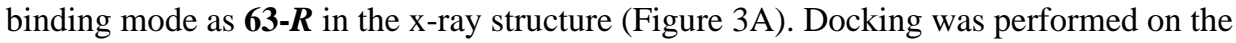
5000 loop models of procaspase- 8 generated in absence of $\mathbf{6 3 - R}$, and the best docking score poses were selected for each molecule. Docking success rates were defined as the percentage of correctly placed rings with an RMSD $\leq 1 \AA$ (calculated on the aromatic ring centroids) from the x-ray coordinates. Consistent with our previous biochemical studies, among all docked ligands, the highest success rate was achieved for $\mathbf{6 3 - R}$, which correctly positioned the phenylamine ring in $24 \%$ of the 5000 models (Figures 6A and B). We had previously found that the enantiomer of 63-R, compound 63-S was nearly ten-fold less potent. ${ }^{30}$ In our docking studies the success rate of $\mathbf{6 3 - S}$ was significantly lower (14\%). For $\mathbf{7}$ and its alkynylated analogue 61, which in ABPP assays, both show similar potency to 63-S the docking success rate was lower ( 6 and $8 \%$, respectively). Gratifyingly, the inactive naphthyl compound 62 shows the lowest success rate with 3\% (Figures 6A and C).

The capability of molecules to dock by engaging the hydrophobic pocket of the phenylamine ring appears to partially recapitulate the trend found in the gel-based ABPP assays (Figure 5). For 62, modeling shows that only a small fraction of the conformationally accessible loop states are compatible with binding, suggesting that the steric clash of the naphthyl group with the hydrophobic cavity near loop 2 is likely the cause of the observed inactivity in ABPP studies (Figure 5). Conversely, when modeling the impact of the W420A mutation, we found that the absence of the aromatic side chain has a detrimental effect on docking accuracy, while no effect was found in the mutagenesis experiments (Figure S3). This result indicates that either procaspase- 8 undergoes a conformational reorganization to compensate for the W420A mutation, or that the W420A substitution might not impact the initial noncovalent interactions and subsequent covalent alkylation of procaspase- 8 by $\mathbf{6 3 - R}$. Also, while experimental data on $\mathbf{6 1}$ and $\mathbf{7}$ show comparable potency with $\mathbf{6 3 - S}$, their docking success rates are smaller than expected (Figures 6D and E). These shortcomings are likely due to the approximations used in the simulations, including the bias of the loop modeling based on the complex with $\mathbf{6 3 - R}$, the protein maintained as rigid during dockings, and docking scoring function limitations. Overall, the docking results show that loop 2 contributes significantly to ligand binding, and our results underscore the general utility of docking studies in characterizing the potential contributions of disordered regions of proteins to molecular recognition of small molecules.

\section{Conclusions}

We report the $\mathrm{x}$-ray co-crystal of procaspase- 8 bound to a non-peptidic small molecule inhibitor 63-R. By integrating X-ray structural data with homology modeling, docking, and focused mutagenesis, we developed a multidisciplinary approach to refine and analyze the structure and mode of inhibition of procaspase-8. Our study builds upon our exciting prior finding that $\mathbf{6 3 - R}$, which selectively targets the pro-form or zymogen of caspase-8, fully protects immortalized lymphocytes from extrinsic (FasL) apoptosis. ${ }^{30}$ The resulting structure-model hybrid revealed the large conformational space of disordered active site loops and rationalized the observed SAR of our compound series. Together, the homology modeling and mutagenesis studies indicate that the flexible active site loops play key roles in recognition of our small molecule inhibitor. Therefore, future structure-guided inhibitor 
design efforts will unquestionably benefit from elucidation of the complete structure of procaspase-8. The current study lays the groundwork for such efforts.

Our study also underscores the need for inhibitors targeting other procaspases. As shown here, such compounds can uniquely enable structural resolution of procaspases. We anticipate that future structural studies leveraging other procaspase inhibitors will yield additional structural insights into this important class of proteins. Caspase-10 is a particularly intriguing candidate for such inhibitor development and structural studies, as no caspase-10 structures are available in the PDB. Given the high sequence homology of caspase- 10 and -8 , homology modeling using our procaspase- 8 structure will likely reveal key features that distinguish caspase- 8 and -10 , including those that can be leveraged to ultimately generate caspase-10-selective inhibitors. Such compounds will enable future studies aimed at deciphering the unique and overlapping functions of caspase- 8 and -10 and will likely prove pivotal for efforts to resolve the structure of procaspase-10.

We also expect that our structure will provide insights into the mechanisms of caspase- 8 activation. Caspase- 8 activation is known to be required for a wide range of important biological processes including canonical roles in lymphocyte activation and FasL-mediated apoptosis as well as key contributions to mammalian development—caspase- 8 is embryonic lethal in rodents. ${ }^{10,37-39}$ Understanding the molecular mechanism of activation will likely guide the design of inhibitors aimed at modulating these processes. Such knowledge should also improve our understanding of the regulatory mechanisms that prevent aberrant activation, including for example interactions with the caspase- 8 decoy protein c-FLIP.

Taken together, the pro-caspase-8 structure, homology modeling, and mutagenesis data presented here lay the foundations for more in-depth studies toward optimized caspase- 8 inhibitors with greater activity and proteome-wide specificity. Such studies will require more extensive biochemical and medicinal chemistry efforts and the application of more extensive computational approaches (e.g., molecular dynamics simulations and free energy calculations) to better capture the structural heterogeneity of the disordered regions of the protein and characterize their role in ligand recognition. More broadly, we anticipate that the integrated approach taken here should readily translate to a wide range of other structures and should prove particularly useful for analysis of both reversibly and irreversibly inhibited protein targets.

\section{Experimental Section}

\section{Procaspase-8 expression and purification.}

Procaspase-8 is over-expressed as a N-terminal His $_{6}$-tag fusion from E. coli BL21DE3pLysS (Strategene) in a pET23b vector. Cells were grown in $2 \times Y T$ media supplemented with 50 $\mu \mathrm{g} / \mathrm{ml}$ ampicillin and chloramphenicol at $37^{\circ} \mathrm{C}$ to an OD600 $\mathrm{nm}$ of 0.6-0.8. Flasks were then transferred to $12{ }^{\circ} \mathrm{C}$ and protein expression was induced with $220 \mu \mathrm{M}$ IPTG for $16 \mathrm{~h}$. Cells were immediately harvested and resuspended in ice cold $100 \mathrm{mM}$ Tris, $\mathrm{pH} 8.0$ and 100 $\mathrm{mM} \mathrm{NaCl}$ (buffer A) and subjected to 3 cycles of lysis by microfluidization (Microfluidics). The cell lysate was clarified by centrifugation at $14,500 \mathrm{x}$ g for $8 \mathrm{~min}$ at $4{ }^{\circ} \mathrm{C}$ and soluble fractions were loaded onto a $5 \mathrm{~mL}$ HisTrap FF crude Ni-NTA affinity column (GE 
Amersham) pre-equilibrated with buffer A and eluted with buffer A containing $250 \mathrm{mM}$ imidazole. The eluted protein was immediately diluted 5-fold with buffer B (20 mM Tris, $\mathrm{pH}$ 8.0) and purified by anion-exchange chromatography (HiTrap Q HP, GE Amersham) with a 20 -column volume gradient to $50 \%$ of buffer B containing $1 \mathrm{M} \mathrm{NaCl}$. Fractions corresponding to procaspase- 8 were pooled and immediately stored at $-80{ }^{\circ} \mathrm{C}$.

\section{Procaspase-8 mutation.}

The expression construct that encodes the $\mathrm{His}_{6}$-tag zymogen form of caspase-8 (residues 214-479 with D374A D384A, C409S, and C433S mutations) to site directed mutagenesis to generate R260A, G418A, C409W, N261A, R258A mutations. Proteins harboring these mutations were expressed and purified from E. coli as described for procaspase-8.

\section{Western blot analysis.}

The concentration of recombinant procaspase- 8 proteins was calculated by NanoDrop ${ }^{\mathrm{TM}}$ and the final protein concentrations adjusted to $500 \mathrm{nM}$ in clarified HEK 293T cellular lysates. The procaspase-containing lysates were then resolved by SDS-PAGE and transferred to PVDF membranes, blocked with 5\% milk in TBST and probed with 6x-His Tag Monoclonal Antibody (HIS.H8) (ThermoFisher MA1-21315, 1:3000). Blots were incubated with primary antibodies overnight at $4{ }^{\circ} \mathrm{C}$ with rocking and were then washed $(3 \times 5 \mathrm{~min}$, TBST) and incubated with secondary antibodies (LICOR, IRDye 800LT goat anti-mouse, 1:10,000) for $1 \mathrm{~h}$ at ambient temperature. Blots were further washed $(3 \times 5 \mathrm{~min}$, TBST) and visualized on a BioRad ChemiDoc ${ }^{\text {TM }}$ MP Imaging System.

\section{LC-MS/MS analysis of procaspase-8 $63-R$ crystals.}

Protein crystals were harvested, washed, and solubilized in $50 \mu \mathrm{L} 8 \mathrm{M}$ urea $(660 \mathrm{mg} / \mathrm{mL}$ in PBS). To this was added $10 \mathrm{mM}$ DTT ( $2.5 \mu \mathrm{L}$ of $200 \mathrm{mM}$ stock solution) and the reaction was incubated at $65^{\circ} \mathrm{C}$ for $15 \mathrm{~min}$ following which $20 \mathrm{mM}$ iodoacetamide $(2.5 \mu \mathrm{L}$ of 400 $\mathrm{mM}$ stock solution) was added and the reaction incubated for $30 \mathrm{~min}$ at $37^{\circ} \mathrm{C}$. The samples were then diluted with $150 \mu \mathrm{L}$ PBS and to this was added $1 \mathrm{mM} \mathrm{CaCl}_{2}(2 \mu \mathrm{L}$ of a $200 \mathrm{mM}$ stock in water) and trypsin ( $2 \mu \mathrm{g}$, Promega, sequencing grade, V5111) and the digestion was allowed to digest overnight at $37{ }^{\circ} \mathrm{C}$ with shaking. The samples were then acidified to a final concentration of $5 \%$ (v/v) formic acid and desalted using C18 Tips (Pierce 87784), following the manufacturer's instructions. The samples were analyzed by liquid chromatography tandem mass spectrometry using an Q Exactive ${ }^{\mathrm{TM}}$ mass spectrometer (Thermo Scientific) coupled to an Easy-nLC ${ }^{\text {TM }} 1000$ pump. The peptides were eluted on a C18 column with a $5 \mu \mathrm{m}$ tip ( $100 \mu \mathrm{m}$ fused silica, $18 \mathrm{~cm})$ using a $140 \mathrm{~min}$ gradient of Buffer B in Buffer A (buffer A: $92 \%$ water, $5 \%$ acetonitrile, $3 \%$ DMSO $0.1 \%$ formic acid; buffer B: $5 \%$ water, $3 \%$ DMSO, $92 \%$ acetonitrile, $0.1 \%$ formic acid) and a flow rate of $200 \mathrm{~nL} / \mathrm{min}$ with electrospray ionization of $2.2 \mathrm{kV}$. Data was collected in data-dependent acquisition mode with dynamic exclusion (15 s) and charge exclusion $(1,7,8,>8)$ enabled. Data acquisition consisted of cycles of one full MS scan $(400-1800 \mathrm{~m} / \mathrm{z}$ at a resolution of 70,000) followed by 12 MS2 scans of the nth most abundant ions at resolution of 17,500. The MS2 spectra data were extracted from the raw file using Raw Converter (version 1.1.0.22; available at http://fields.scripps.edu/rawconv/). MS2 spectra were searched using the ProLuCID algorithm (publicly available at http://fields.scripps.edu/yates/wp/?page_id=17) 
using a reverse concatenated and nonredundant variant of the Human UniProt database (release-2012_11) modified to include the sequence of caspase-8 harboring D374A D384A C409S C433S point mutations. Cysteine residues were searched with a static modification for carboxyamidomethylation $(+57.02146 \mathrm{C})$. Searches also included methionine oxidation as a differential modification $(+15.9949 \mathrm{M})$ and labeling by the compound $\mathbf{6 3 - R}$ $(+348.18378 \mathrm{C})$ as a differential modification. ${ }^{40}$

\section{Circular dichroism.}

Circular dichroism (CD) was measured in a JASCO J-715 CD spectrophotometer, scanning 2 times from $250-195 \mathrm{~nm}$ at $50 \mathrm{~nm} / \mathrm{min}$, time constant $=4 \mathrm{sec}$, bandwidth $=1 \mathrm{~nm}$, slit width $=500 \mu \mathrm{m} .0 .3 \mathrm{mg} / \mathrm{mL}$ protein solutions in buffer $(25 \mathrm{mM}$ Tris $\mathrm{HCl}, \mathrm{pH} 7.4$ and 8.3 $\mathrm{mM} \mathrm{NaCl}$ ) were held in $0.1 \mathrm{~cm}$ path length quartz cuvettes. Each secondary structure data set was analyzed via SELCON method against Hennessy and Johnson reference proteins. 4142

\section{Crystallization and $\mathrm{x}$-ray data collection.}

Inhibitor 63- $\boldsymbol{R}$ was added in a 3-fold molar excess to procaspase-8 (300 uM) in $20 \mathrm{mM}$ Tris, pH 8.0 and $10 \mathrm{mM}$ DTT. The protein:inhibitor mixture was clarified of any precipitant by centrifugation at $3000 \mathrm{x}$ g for $3 \mathrm{~min}$. Crystals were grown by sitting drop-vapor diffusion by mixing equal volumes $(1.5 \mu \mathrm{l})$ of the procaspase-8:63- $R$ complex and reservoir solution consisting of $0.08 \mathrm{M}$ imidazole, $\mathrm{pH} 8.0$ and $1 \mathrm{M}$ sodium citrate at $25^{\circ} \mathrm{C}$. Data was collected on a single, flash-cooled crystal at $100 \mathrm{~K}$ in a cryoprotectant consisting of mother liquor and $25 \%$ glycerol and were processed with HKL2000 in orthorhombic space group P $3_{1}$. The calculated Matthews' coefficient $\left(\mathrm{V}_{\mathrm{M}}=3.14 \AA^{3} \mathrm{Da}^{-1}\right)$ suggested six monomers per asymmetric unit with a solvent content of $60 \%$. X-ray data was collected to $2.88 \AA$ resolution on beamline 9.2 at the Stanford Synchrotron Radiation Lightsource (SSRL) (Menlo Park, CA).

\section{Structure solution and refinement.}

The procaspase- 8 structure was determined by molecular replacement (MR) with Phaser $^{43}$ using the previously published active caspase structure (PDB 4JJ7) as the initial search model. The structure was manually built with WinCoot ${ }^{44}$ and iteratively refined using Phenix ${ }^{45}$ with cycles of conventional positional refinement with isotropic B-factor refinement. Non-crystallographic symmetry (NCS) constraints were applied. The electron density maps clearly identified that $\mathbf{6 3 -} \boldsymbol{R}$ was covalently attached to Cys360 within the active site in subunit B. Water molecules were automatically positioned by Phenix using a $2.5 \sigma$ cutoff in $f_{\mathrm{o}}-f_{\mathrm{c}}$ maps and manually inspected. The final $\mathrm{R}_{\text {cryst }}$ and $\mathrm{R}_{\text {free }}$ are $28.9 \%$ and $36.6 \%$, respectively (Figure 2 and Table $S 1$ ). The model was analyzed and validated with the PDB Validation Server prior to PDB deposition. Analysis of backbone dihedral angles with the program PROCHECK ${ }^{46}$ indicated that all residues are located in the most favorable and additionally allowed regions in the Ramachandran plot. Coordinates and structure factors have been deposited in the PDB with accession entry 6PX9. Structure refinement statistics are shown in Supplementary Table 1. 


\section{Loop modeling and analysis.}

Several models of the missing N-terminal residues 217-222, as well as the missing residues in loops 1, 2, and 3, were built using MODELLER 9v21. ${ }^{47}$ The homodimeric crystal structure of procaspase- 8 subunits A and B within the asymmetric unit with covalently bound $\mathbf{6 3 - R}$ to C360 in chain B was used as the structural template. Due to the lack of density for the side chain of R258 (chain B) and its apparent proximity with the N-terminal region of the flexible loop 2, the side chain orientation was also refined during the loop modeling. Each model was first optimized twice with the variable target function method (VTFM) set to the slow level with 300 steps of conjugate gradients (CG) and an objective function cutoff of $1 \times 10^{6}$. The models were subsequently refined using molecular dynamics (MD) coupled with simulated annealing (SA), set at the slow level. This protocol was applied to generate 5,000 models of the homodimer consisting of chains A and B.

For the analysis, models were ranked by using the DOPE energy score from MODELLER, and the top 1,000 results were selected for $\mathbf{6 3}-\boldsymbol{R}$ binding analysis. Using the Python module MDAnalysis ${ }^{48}$ the occupancy of each residue in close contact with the covalently bound $\mathbf{6 3 -}$ $\boldsymbol{R}$, using a distance cutoff of $4 \AA$, was computed from the selected models. The occupancy was defined as the ratio of the number of models where the residue $i$ is in close contact ( $\leq 4$ $\AA$ ) with the ligand over the total number of selected models. The occupancy values are ranging from $0 \%$ (never in close contact) to $100 \%$ (always in contact). To increase resolution, backbone and sidechain of each residue were considered independently.

\section{Molecular docking.}

Using the previously described loop modeling protocol, 5000 additional models were generated in the absence of ligand in order to limit biases toward 63- $R$. In contrast with the loop analysis, all models were used for the docking of $7,61,62,63-R$, and $63-S$. The models were prepared for docking by adding hydrogen atoms with REDUCE ${ }^{49}$ at $\mathrm{pH} 7.0$ with Asn, Gln and His sidechains allowed to flip, then following the standard preparation protocol. ${ }^{50}$ The 3D coordinates of compound $\mathbf{6 3}-\boldsymbol{R}$ were taken from the crystallographic structure of procaspase- 8 , and compounds $7,61,62$, and $63-S$ were built with the builder module in PyMOL, ${ }^{51}$ using $\mathbf{6 3 -} \boldsymbol{R}$ as reference. The affinity maps were generated using AutoGrid with the standard AutoDock forcefield. The center of the search space was set to position $\mathrm{x}$ : $-14.0, y:-22.0, z: 10$ ), the size of the grid set to $60 \times 60 \times 60$ with a grid spacing of $0.375 \AA$ and the smoothing was removed. The ligands were then prepared for covalent docking for AutoDock 4.2.1 52 on Cys 360 following the flexible residue protocol. ${ }^{53}$ The standard GA parameters were used to generate 10 docked poses, and the lowest energy pose was selected for each docking.

\section{Gel-based activity-based protein profiling.}

$25 \mu \mathrm{L}$ of soluble proteome $(1 \mathrm{mg} / \mathrm{mL}$ ) containing procaspase- 8 ( $500 \mathrm{nM}$ each respectively) was labeled with the indicated concentration of the indicated compounds $(1 \mu \mathrm{L}$ of $25 \times$ stock solution in DMSO) for $1 \mathrm{~h}$ at ambient temperature followed by labeling with $10 \mu \mathrm{M}$ of probe $61(1 \mu \mathrm{L}$ of $25 \times$ stock solution in DMSO). Subsequently, the samples were subjected to $\mathrm{CuAAC}$ conjugation to rhodamine-azide for $1 \mathrm{~h}$ at ambient temperature. CuAAC was performed with $20 \mu \mathrm{M}$ rhodamine-azide (50x stock in DMSO), $1 \mathrm{mM}$ tris(2- 
carboxyethyl)phosphine hydrochloride (TCEP; fresh 50x stock in water, final concentration $=1 \mathrm{mM})$, ligand $(17 \times$ stock in DMSO:t-butanol 1:4, final concentration $=100 \mu \mathrm{M})$ and 1 $\mathrm{mM} \mathrm{CuSO}_{4}(50 \times$ stock in water, final concentration $=1 \mathrm{mM})$. Samples were quenched with $10 \mu \mathrm{L} 4 \times$ SDS-PAGE loading buffer. Quenched reactions were analyzed by SDS-PAGE and visualized by in-gel fluorescence.

\title{
Determination of apparent $I_{50}$ values.
}

$25 \mu \mathrm{L}$ of proteomes containing the indicated protein at $500 \mathrm{nM}$ final concentration were treated with the indicated compounds for $1 \mathrm{~h}$ at ambient temperature, labeled with probe $\mathbf{6 1}$ for $1 \mathrm{~h}$, subjected to CuAAC conjugation to rhodamine-azide, quenched, and analyzed by SDS-PAGE and in-gel fluorescence visualization $(n=3)$. The percentage of labeling was determined by quantifying the integrated optical intensity of the bands, using ImageJ software 14. Nonlinear regression analysis was used to determine the apparent $\mathrm{IC}_{50}$ values from a dose-response curve generated using GraphPad Prism 6.

\section{Statistical analysis.}

Data are shown as mean $\pm \mathrm{SD}$. $P$-values were calculated using unpaired, two-tailed Student's t-test with values $<0.05$ considered significant.

\section{Supplementary Material}

Refer to Web version on PubMed Central for supplementary material.

\section{ACKNOWLEDGMENT}

\author{
We thank I. Wilson, R. Stanfield, M. Elsliger, and X. Dai for computational assistance, H. Rosen for access to \\ instrumentation, and the staff of the Stanford Synchrotron Radiation Lightsource. \\ Funding Sources \\ This work was supported by the National Institutes of Health R01GM118382 (to DWW) and R01GM069832 (to \\ SF), and the Department of Energy DE-FC02-02ER63421 (to KMB).
}

\section{ABBREVIATIONS}
ABPP
activity-based protein profiling
CD
circular dichroism
CI
confidence interval
CuAAC
$\mathrm{Cu}(\mathrm{I})$-catalyzed azide-alkyne cycloaddition
DMSO
dimethyl sulfoxide
DTT
dithiothreitol
IPTG
isopropyl- $\beta$-D-thiogalactoside
LC-MS/MS
liquid chromatography tandem mass spectrometry 
NMR nuclear magnetic resonance

RMSD root-mean-square-deviation

SAR structure activity relationship

SD standard deviation

SDS-PAGE sodium dodecyl sulfate-polyacrylamide gel electrophoresis

\section{REFERENCES}

1. Shalini S, Dorstyn L, Dawar S, and Kumar S (2015) Old, new and emerging functions of caspases, Cell Death Differ. 22, 526-539. [PubMed: 25526085]

2. Li J, and Yuan J (2008) Caspases in apoptosis and beyond, Oncogene 27, 6194-6206. [PubMed: 18931687]

3. Solier S, Fontenay M, Vainchenker W, Droin N, and Solary E (2017) Non-apoptotic functions of caspases in myeloid cell differentiation, Cell Death Differ. 24, 1337-1347. [PubMed: 28211870]

4. Fogarty CE, and Bergmann A (2017) Killers creating new life: caspases drive apoptosis-induced proliferation in tissue repair and disease, Cell Death Differ. 24, 1390-1400. [PubMed: 28362431]

5. Mukhopadhya I, Hansen R, El-Omar EM, and Hold GL (2012) IBD: what role do Proteobacteria play?, Nat. Rev. Gastroenterol. Hepatol. 9, 219-230. [PubMed: 22349170]

6. Nambiar PR, Gupta RR, and Misra V (2010) An "Omics” based survey of human colon cancer, Mutat. Res. 693, 3-18. [PubMed: 20691711]

7. Olsson M, and Zhivotovsky B (2011) Caspases and cancer, Cell Death Differ. 18, 1441-1449. [PubMed: 21455218]

8. Troy CM, and Jean YY (2015) Caspases: therapeutic targets in neurologic disease, Neurotherapeutics 12, 42-48. [PubMed: 25339539]

9. Rehker J, Rodhe J, Nesbitt RR, Boyle EA, Martin BK, Lord J, Karaca I, Naj A, Jessen F, Helisalmi S, Soininen H, Hiltunen M, Ramirez A, Scherer M, Farrer LA, Haines JL, Pericak-Vance MA, Raskind WH, Cruchaga C, Schellenberg GD, Joseph B, and Brkanac Z (2017) Caspase-8, association with Alzheimer's disease and functional analysis of rare variants, PLoS One 12, e0185777.

10. Su H, Bidere N, Zheng L, Cubre A, Sakai K, Dale J, Salmena L, Hakem R, Straus S, and Lenardo $\mathrm{M}$ (2005) Requirement for caspase- 8 in NF- $\kappa$ B activation by antigen receptor, Science $307,1465-$ 1468. [PubMed: 15746428]

11. Man SM, and Kanneganti TD (2016) Converging roles of caspases in inflammasome activation, cell death and innate immunity, Nat. Rev. Immunol. 16, 7-21. [PubMed: 26655628]

12. Cullen SP, and Martin SJ (2009) Caspase activation pathways: some recent progress, Cell Death Differ. 16, 935-938. [PubMed: 19528949]

13. Johnson CE, and Kornbluth S (2008) Caspase cleavage is not for everyone, Cell 134, 720-721. [PubMed: 18775303]

14. Poreba M, Szalek A, Kasperkiewicz P, Rut W, Salvesen GS, and Drag M (2015) Small molecule active site directed tools for studying human caspases, Chem. Rev. 115, 12546-12629. [PubMed: 26551511]

15. Vickers CJ, Gonzalez-Paez GE, and Wolan DW (2013) Selective detection and inhibition of active caspase-3 in cells with optimized peptides, J. Am. Chem. Soc. 135, 12869-12876. [PubMed: 23915420]

16. Julien O, and Wells JA (2017) Caspases and their substrates, Cell Death Differ. 24, 1380-1389. [PubMed: 28498362]

17. Thornberry NA, Rano TA, Peterson EP, Rasper DM, Timkey T, Garcia-Calvo M, Houtzager VM, Nordstrom PA, Roy S, Vaillancourt JP, Chapman KT, and Nicholson DW (1997) A combinatorial approach defines specificities of members of the caspase family and granzyme B. Functional 
relationships established for key mediators of apoptosis, J. Biol. Chem. 272, 17907-17911. [PubMed: 9218414]

18. Hardy JA, Lam J, Nguyen JT, O'Brien T, and Wells JA (2004) Discovery of an allosteric site in the caspases, Proc. Natl. Acad. Sci. USA 101, 12461-12466. [PubMed: 15314233]

19. Scheer JM, Romanowski MJ, and Wells JA (2006) A common allosteric site and mechanism in caspases, Proc. Natl. Acad. Sci. USA 103, 7595-7600. [PubMed: 16682620]

20. Edgington LE, van Raam BJ, Verdoes M, Wierschem C, Salvesen GS, and Bogyo M (2012) An optimized activity-based probe for the study of caspase-6 activation, Chem. Biol. 19, 340-352. [PubMed: 22444589]

21. Boatright KM, Renatus M, Scott FL, Sperandio S, Shin H, Pedersen IM, Ricci JE, Edris WA, Sutherlin DP, Green DR, and Salvesen GS (2003) A unified model for apical caspase activation, Mol. Cell. 11, 529-541. [PubMed: 12620239]

22. Boatright KM, and Salvesen GS (2003) Mechanisms of caspase activation, Curr. Opin. Cell Biol. 15, 725-731. [PubMed: 14644197]

23. Chai J, Wu Q, Shiozaki E, Srinivasula SM, Alnemri ES, and Shi Y (2001) Crystal structure of a procaspase-7 zymogen: mechanisms of activation and substrate binding, Cell 107, 399-407. [PubMed: 11701129]

24. Thomsen ND, Koerber JT, and Wells JA (2013) Structural snapshots reveal distinct mechanisms of procaspase-3 and -7 activation, Proc. Natl. Acad. Sci. USA 110, 8477-8482. [PubMed: 23650375]

25. Elliott JM, Rouge L, Wiesmann C, and Scheer JM (2009) Crystal structure of procaspase-1 zymogen domain reveals insight into inflammatory caspase autoactivation, J. Biol. Chem. 284, 6546-6553. [PubMed: 19117953]

26. Murray J, Giannetti AM, Steffek M, Gibbons P, Hearn BR, Cohen F, Tam C, Pozniak C, Bravo B, Lewcock J, Jaishankar P, Ly CQ, Zhao X, Tang Y, Chugha P, Arkin MR, Flygare J, and Renslo AR (2014) Tailoring small molecules for an allosteric site on procaspase-6, ChemMedChem 9, 73-77, 72. [PubMed: 24259468]

27. Keller N, Mares J, Zerbe O, and Grutter MG (2009) Structural and biochemical studies on procaspase-8: new insights on initiator caspase activation, Structure 17, 438-448. [PubMed: 19278658]

28. Pop C, Feeney B, Tripathy A, and Clark AC (2003) Mutations in the procaspase-3 dimer interface affect the activity of the zymogen, Biochemistry 42, 12311-12320. [PubMed: 14567692]

29. Philip NH, DeLaney A, Peterson LW, Santos-Marrero M, Grier JT, Sun Y, Wynosky-Dolfi MA, Zwack EE, Hu B, Olsen TM, Rongvaux A, Pope SD, Lopez CB, Oberst A, Beiting DP, HenaoMejia J, and Brodsky IE (2016) Activity of uncleaved caspase- 8 controls anti-bacterial immune defense and TLR-induced cytokine production independent of cell death, PLoS Pathog. 12, e1005910.

30. Backus KM, Correia BE, Lum KM, Forli S, Horning BD, Gonzalez-Paez GE, Chatterjee S, Lanning BR, Teijaro JR, Olson AJ, Wolan DW, and Cravatt BF (2016) Proteome-wide covalent ligand discovery in native biological systems, Nature 534, 570-574. [PubMed: 27309814]

31. Medema JP, Scaffidi C, Kischkel FC, Shevchenko A, Mann M, Krammer PH, and Peter ME (1997) FLICE is activated by association with the CD95 death-inducing signaling complex (DISC), EMBO J. 16, 2794-2804. [PubMed: 9184224]

32. Engels IH, Stepczynska A, Stroh C, Lauber K, Berg C, Schwenzer R, Wajant H, Janicke RU, Porter AG, Belka C, Gregor M, Schulze-Osthoff K, and Wesselborg S (2000) Caspase-8/FLICE functions as an executioner caspase in anticancer drug-induced apoptosis, Oncogene 19, 45634573. [PubMed: 11030145]

33. Nagar B, Bornmann WG, Pellicena P, Schindler T, Veach DR, Miller WT, Clarkson B, and Kuriyan $\mathrm{J}$ (2002) Crystal structures of the kinase domain of c-Abl in complex with the small molecule inhibitors PD173955 and imatinib (STI-571), Cancer Res. 62, 4236-4243. [PubMed: 12154025]

34. Capdeville R, Buchdunger E, Zimmermann J, and Matter A (2002) Glivec (STI571, imatinib), a rationally developed, targeted anticancer drug, Nat. Rev. Drug. Discov. 1, 493-502. [PubMed: 12120256]

35. van Montfort RL, and Workman P (2009) Structure-based design of molecular cancer therapeutics, Trends Biotechnol. 27, 315-328. [PubMed: 19339067] 
36. Lin YL, Meng Y, Jiang W, and Roux B (2013) Explaining why Gleevec is a specific and potent inhibitor of Abl kinase, Proc. Natl. Acad. Sci. USA 110, 1664-1669. [PubMed: 23319661]

37. Chun HJ, Zheng L, Ahmad M, Wang J, Speirs CK, Siegel RM, Dale JK, Puck J, Davis J, Hall CG, Skoda-Smith S, Atkinson TP, Straus SE, and Lenardo MJ (2002) Pleiotropic defects in lymphocyte activation caused by caspase- 8 mutations lead to human immunodeficiency, Nature 419, 395-399. [PubMed: 12353035]

38. Salmena L, Lemmers B, Hakem A, Matysiak-Zablocki E, Murakami K, Au PY, Berry DM, Tamblyn L, Shehabeldin A, Migon E, Wakeham A, Bouchard D, Yeh WC, McGlade JC, Ohashi PS, and Hakem R (2003) Essential role for caspase 8 in T-cell homeostasis and T-cell-mediated immunity, Genes Dev. 17, 883-895. [PubMed: 12654726]

39. Varfolomeev EE, Schuchmann M, Luria V, Chiannilkulchai N, Beckmann JS, Mett IL, Rebrikov D, Brodianski VM, Kemper OC, Kollet O, Lapidot T, Soffer D, Sobe T, Avraham KB, Goncharov T, Holtmann H, Lonai P, and Wallach D (1998) Targeted disruption of the mouse caspase 8 gene ablates cell death induction by the TNF receptors, Fas/Apo1, and DR3 and is lethal prenatally, Immunity 9, 267-276. [PubMed: 9729047]

40. Tabb DL, McDonald WH, and Yates JR 3rd. (2002) DTASelect and Contrast: tools for assembling and comparing protein identifications from shotgun proteomics, J. Proteome Res. 1, 21-26. [PubMed: 12643522]

41. Sreerama N, and Woody RW (1993) A self-consistent method for the analysis of protein secondary structure from circular dichroism, Anal. Biochem. 209, 32-44. [PubMed: 8465960]

42. Hennessey JP Jr., and Johnson WC Jr. (1981) Information content in the circular dichroism of proteins, Biochemistry 20, 1085-1094. [PubMed: 7225319]

43. McCoy AJ, Grosse-Kunstleve RW, Adams PD, Winn MD, Storoni LC, and Read RJ (2007) Phaser crystallographic software, J. Appl. Crystallogr. 40, 658-674. [PubMed: 19461840]

44. Emsley P, Lohkamp B, Scott WG, and Cowtan K (2010) Features and development of Coot, Acta Crystallogr. D Biol. Crystallogr. 66, 486-501. [PubMed: 20383002]

45. Adams PD, Afonine PV, Bunkoczi G, Chen VB, Davis IW, Echols N, Headd JJ, Hung LW, Kapral GJ, Grosse-Kunstleve RW, McCoy AJ, Moriarty NW, Oeffner R, Read RJ, Richardson DC, Richardson JS, Terwilliger TC, and Zwart PH (2010) PHENIX: a comprehensive Python-based system for macromolecular structure solution, Acta Crystallogr. D Biol. Crystallogr. 66, 213-221. [PubMed: 20124702]

46. Laskowski RA, Macarthur MW, Moss DS, and Thornton JM (1993) Procheck - a program to check the stereochemical quality of protein structures, J. Appl. Crystallogr. 26, 283-291.

47. Webb B, and Sali A (2014) Protein structure modeling with MODELLER, pp 1-15, Humana Press, New York, NY, New York, NY.

48. Michaud-Agrawal N, Denning EJ, Woolf TB, and Beckstein O (2011) MDAnalysis: a toolkit for the analysis of molecular dynamics simulations, J. Comput. Chem. 32, 2319-2327. [PubMed: 21500218]

49. Word JM, Lovell SC, Richardson JS, and Richardson DC (1999) Asparagine and glutamine: using hydrogen atom contacts in the choice of side-chain amide orientation, J. Mol. Biol. 285, 17351747. [PubMed: 9917408]

50. Forli S, Huey R, Pique ME, Sanner MF, Goodsell DS, and Olson AJ (2016) Computational protein-ligand docking and virtual drug screening with the AutoDock suite, Nat. Protoc. 11, 905919. [PubMed: 27077332]

51. Lill MA, and Danielson ML (2011) Computer-aided drug design platform using PyMOL, J. Comput. Aided Mol. Des. 25, 13-19. [PubMed: 21053052]

52. Morris GM, Huey R, Lindstrom W, Sanner MF, Belew RK, Goodsell DS, and Olson AJ (2009) AutoDock4 and AutoDockTools4: automated docking with selective receptor flexibility, J. Comput. Chem. 30, 2785-2791. [PubMed: 19399780]

53. Bianco G, Forli S, Goodsell DS, and Olson AJ (2016) Covalent docking using AutoDock: twopoint attractor and flexible side chain methods, Protein Sci. 25, 295-301. [PubMed: 26103917] 

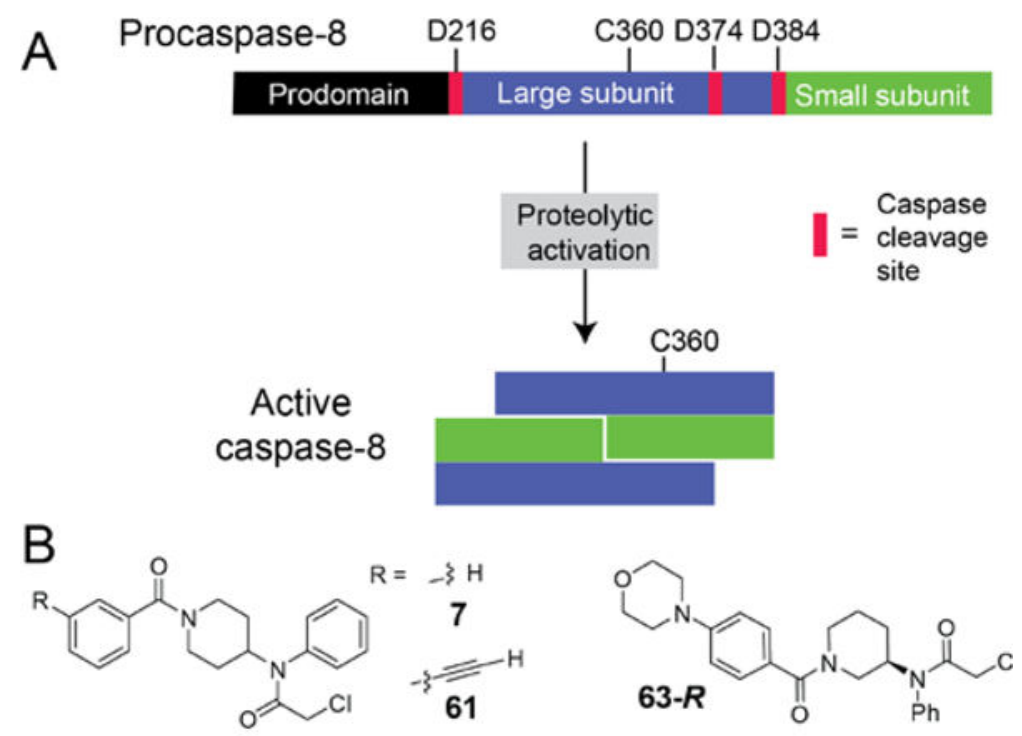

Dual procaspase-8/10

Procaspase- 8 selective

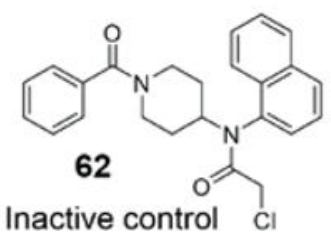

Figure 1.

Caspase activation and structures of procaspase inhibitors. (A) General scheme for activation of procaspase- 8 by proteolysis after conserved aspartate residues. (B) The structures of caspase-8 lead compounds 7 and $\mathbf{6 3 - R}$, alkyne-containing chemical probe $\mathbf{6 1}$ and inactive control compound $\mathbf{6 2} .{ }^{30}$ 

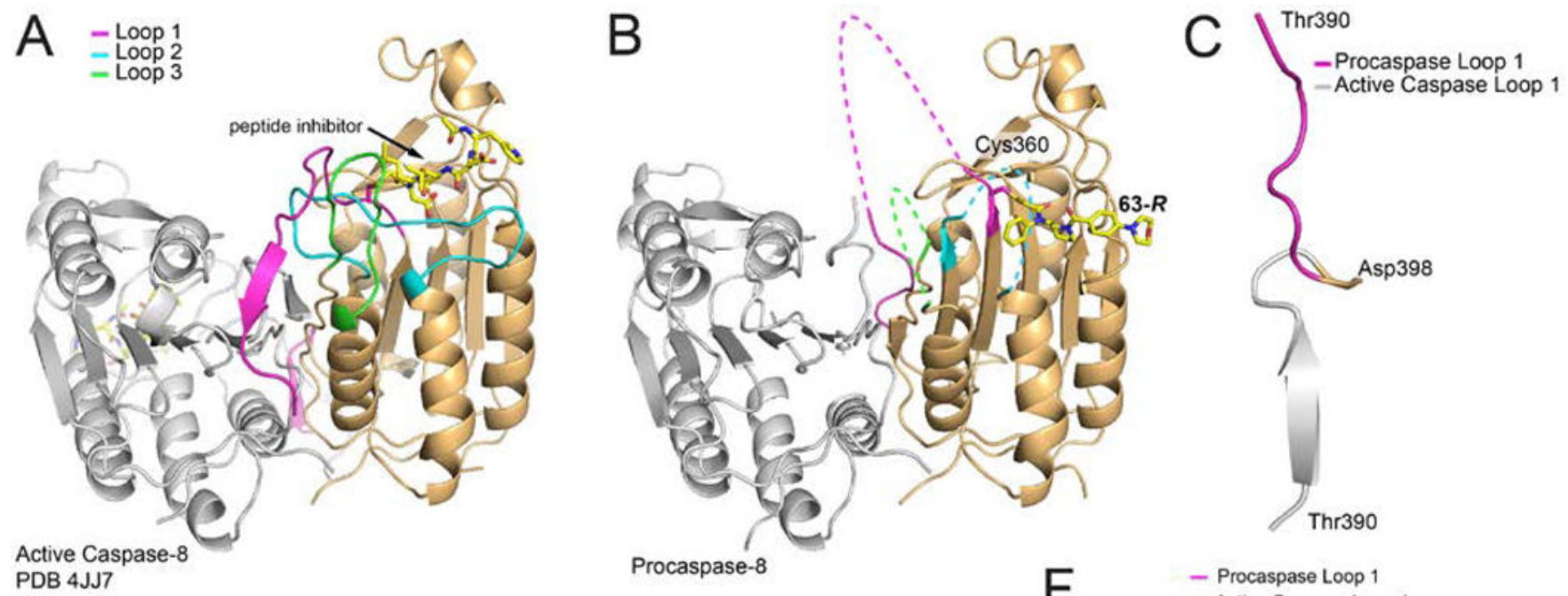

D

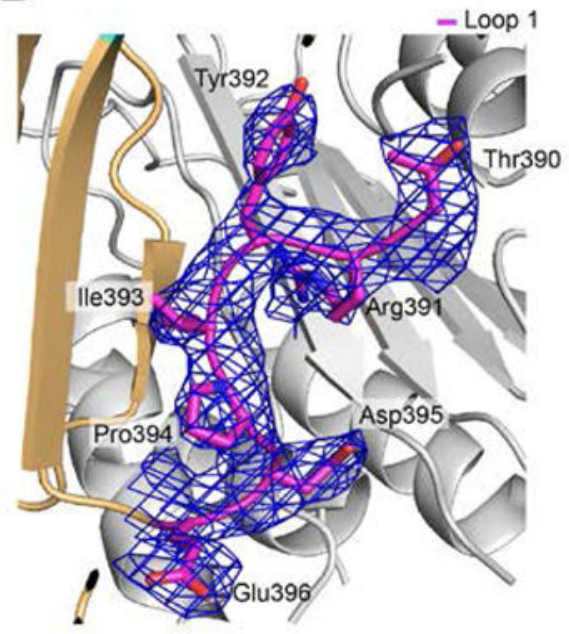

$$
\text { Procaspase-8 }
$$$$
\text { - Subunit A }
$$

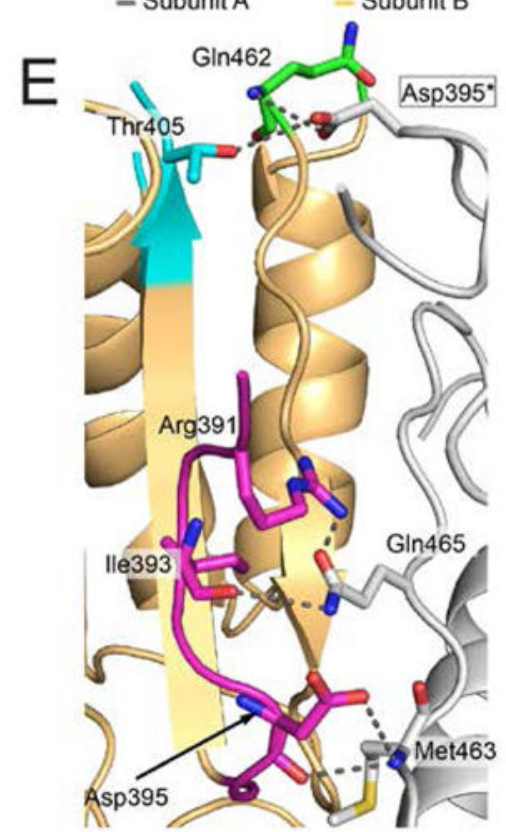

F
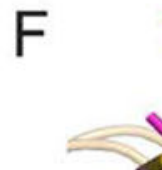

- Procaspase Loop 1 - Active Caspase Loop

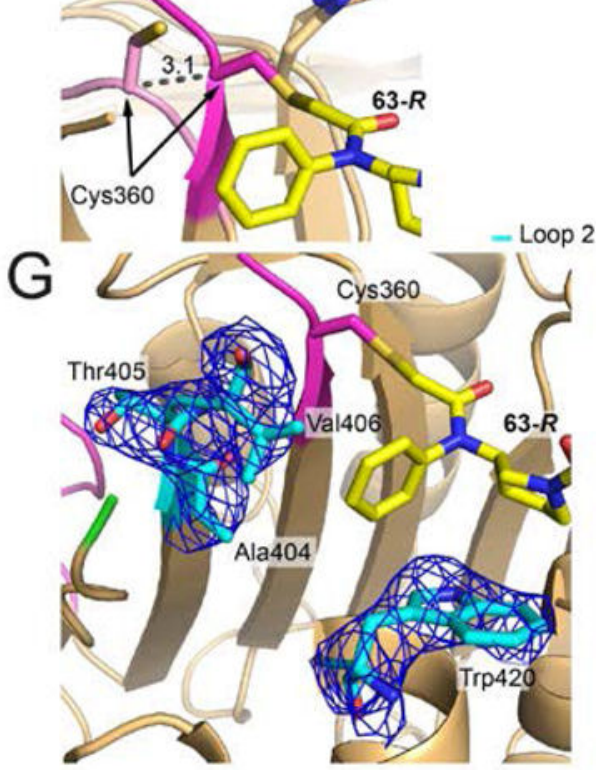

Figure 2.

Crystal structure of human procaspase-8. (A) Cartoon representation of homodimeric active caspase- 8 bound to covalent inhibitor, Ac-3Pal-D- $\beta$ hLeu-hLeu-D-AOMK (yellow) shown with the catalytic cysteine (Cys 360) highlighted in magenta and the start and end residues of the three disordered loops, loop 1 (359-396), loop 2, (404-420) and loop 3 (452-462) highlighted in magenta, cyan, and green, respectively, with individual subunits colored tan and grey. (B) The structure of homodimeric procaspase- 8 with one chain bound to covalent inhibitor, 63-R. Loops, catalytic cysteine, and inhibitor are colored as in 'A'. (C) Overlay of the C-terminal end of loop 1 in active caspase-8 (grey) and procaspase-8 (magenta). Release of loop 1 due to cleavage of the activation linker results in a $180^{\circ}$ flip relative to the position of loop 1 in procaspase-8. (D) $2 f_{\mathrm{O}}-f_{\mathrm{c}}$ density map of well-positioned C-terminal end of loop 1 contoured at $1.0 \sigma$ is shown in blue mesh. (E) Potential hydrogen bonds between loops 1, 2 , and 3 and the partnering chain at the dimer interface with colors conserved in ' $A$ '. (F) 
Overlay of the catalytic cysteine in active caspase- 8 (pink) and procaspase- 8 (magenta). (G) $2 f_{\mathrm{O}}-f_{\mathrm{c}}$ density map of loop 2 contoured at $1.0 \sigma$ as in 'D'. 

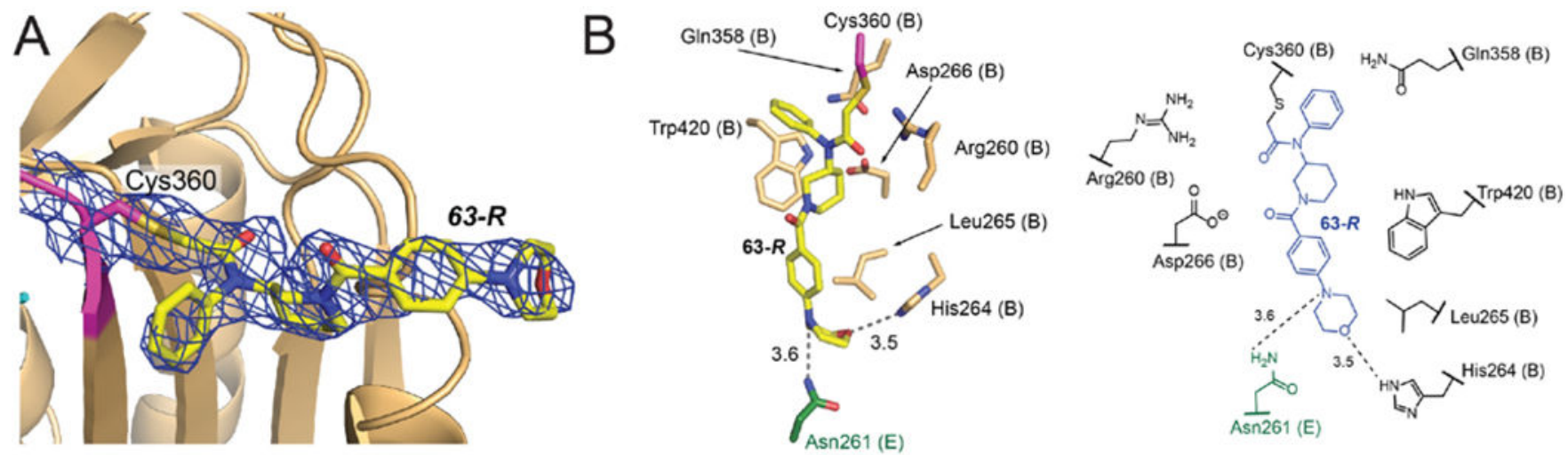

Figure 3.

Procaspase- 8 in complex with covalent inhibitor 63-R. (A) $2 f_{0}-f_{\mathrm{c}}$ density map of 63- $\boldsymbol{R}$ bound to catalytic Cys360 contoured at $1.0 \sigma$ shown in blue mesh. (B) Schematic showing the interactions between procaspase- 8 and $\mathbf{6 3 - R}$ (yellow carbon in the left stick model and blue in the right schematic). Aside from potential hydrogen bonds between $\mathbf{6 3 - R}$ and His264 as well as a crystal contact from Asn261 of subunit E (green carbon), the rest of the residues form hydrophobic interactions with the inhibitor. 


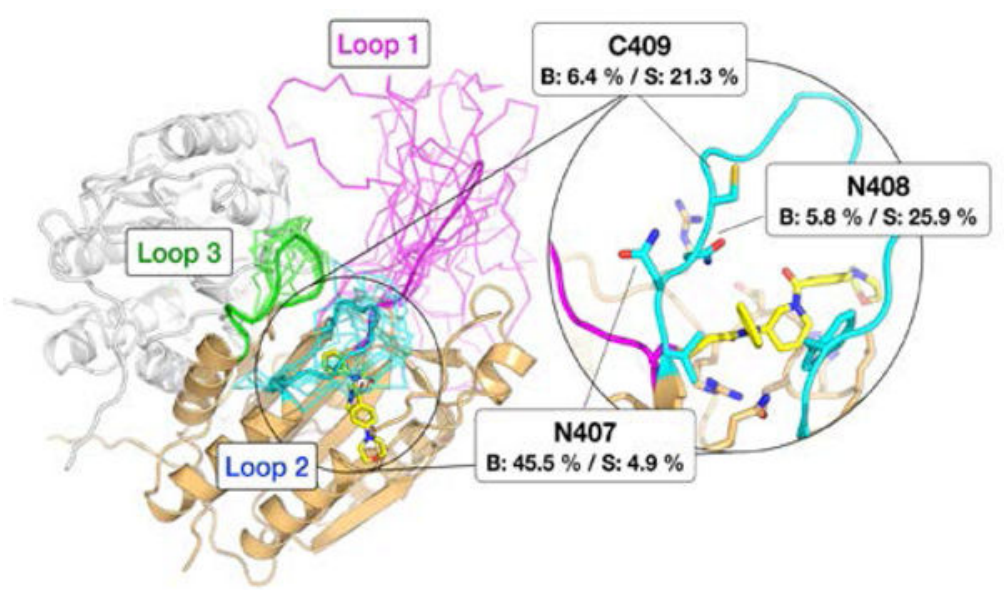

Figure 4.

Models of missing loops of procaspase-8 in complex with covalent inhibitor 63-R. Ribbon representation of the 10 best models, generated with MODELLER, are shown with the start and end residues of the three disordered loops, loop 1 (359-396), loop 2, (404-420) and loop 3 (452-462) colored in magenta, cyan, and green, respectively, with individual subunits in tan and grey. In cartoon representation and as stick, the representative model of the three

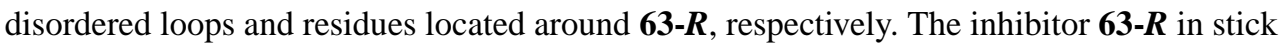
representation with yellow carbon is shown with the backbone (B) and side-chain (S) occupancies of residues N407, N408 and C409 of procaspase-8, as well as the representative model of the loops obtained from the 1000 best models. 

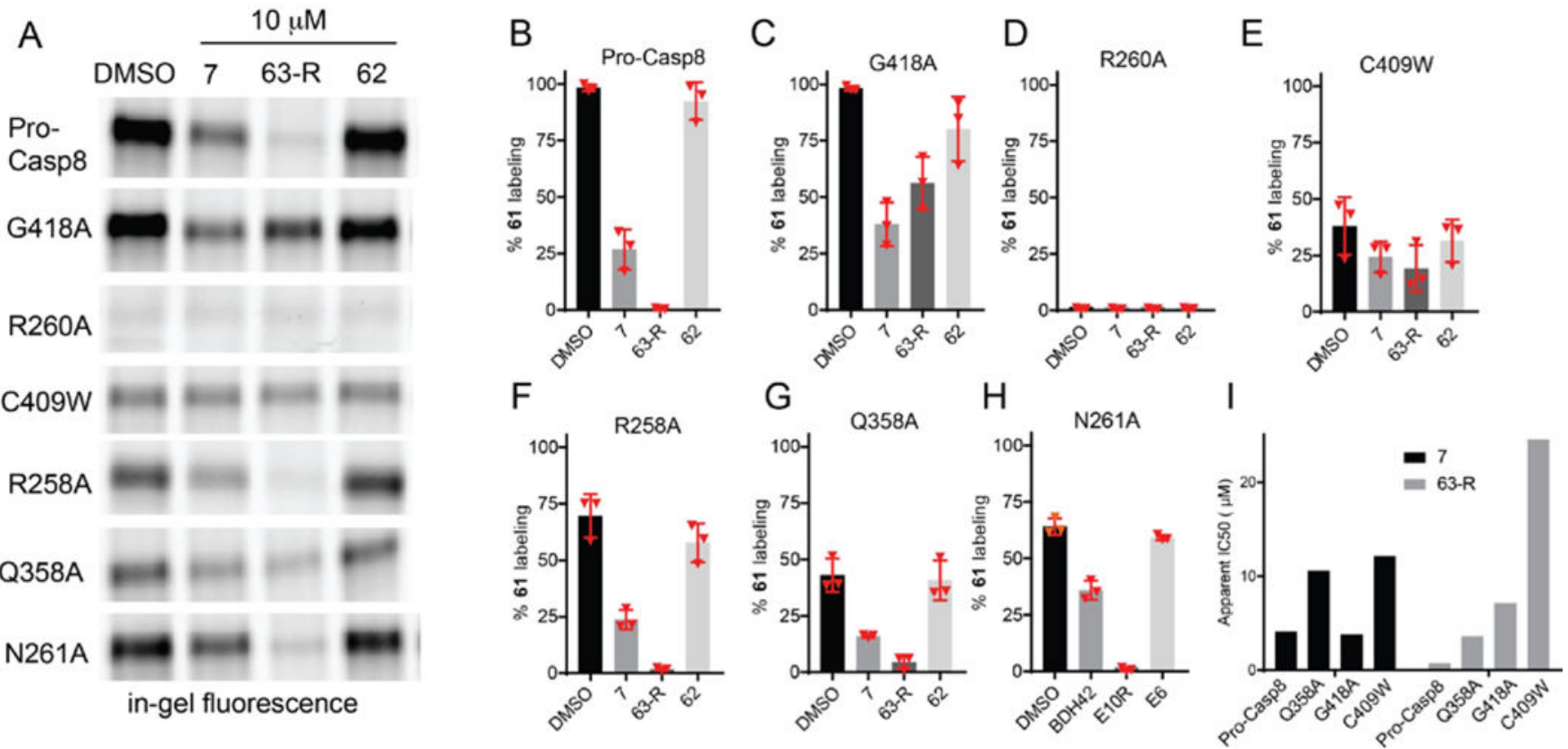

Figure 5.

Mutational studies to identify residues that alter probe labeling of procaspase-8. (A)

Procaspase-8 recombinant protein (D384A and D394A to prevent activation), which also harbor the indicated mutated residues, were evaluated by gel-based ABPP. Equal concentrations of individual proteins were added to HEK 293T cellular lysates, treated with $\mathbf{7}, \mathbf{6 3}-\boldsymbol{R}, \mathbf{6 2}$, or vehicle (DMSO) for 1h, followed by labeling with $61(10 \mu \mathrm{M})$ for $1 \mathrm{~h}$, "click" conjugation to rhodamine-azide and analysis by SDS-PAGE and in-gel fluorescence.

Decrease in fluorescence intensity indicates competition of $\mathbf{6 1}$ labeling by compound pretreatment. (B-H) Quantification of the gel-based data shown in 'B'. The integrated fluorescence band intensities were quantified and the percentage labeling by $\mathbf{6 1}$ was calculated relative to the integrated intensity of DMSO-treated procaspase-8. Shown are the mean and standard deviations derived from three replicate independent experiments for (B) procaspase-8, (C) G418A, (D) R260A, (E) C409W, (F) R258A, (G) Q358A, (H) N261A. (I) For the mutant proteins that showed significantly altered compound labeling in 'B-C' the apparent half maximal inhibitory concentration $\left(\mathrm{IC}_{50}\right.$ ) values for compounds $\mathbf{7}$ and $\mathbf{6 3 - \boldsymbol { R }}$ were calculated for blockade of 61-labeling from the mean $+/-$ SD of triplicate experiments. Quantification of the decrease in fluorescence compared to vehicle treated samples was calculated from the total integrated intensity of the labeled bands for procaspase- 8 . 

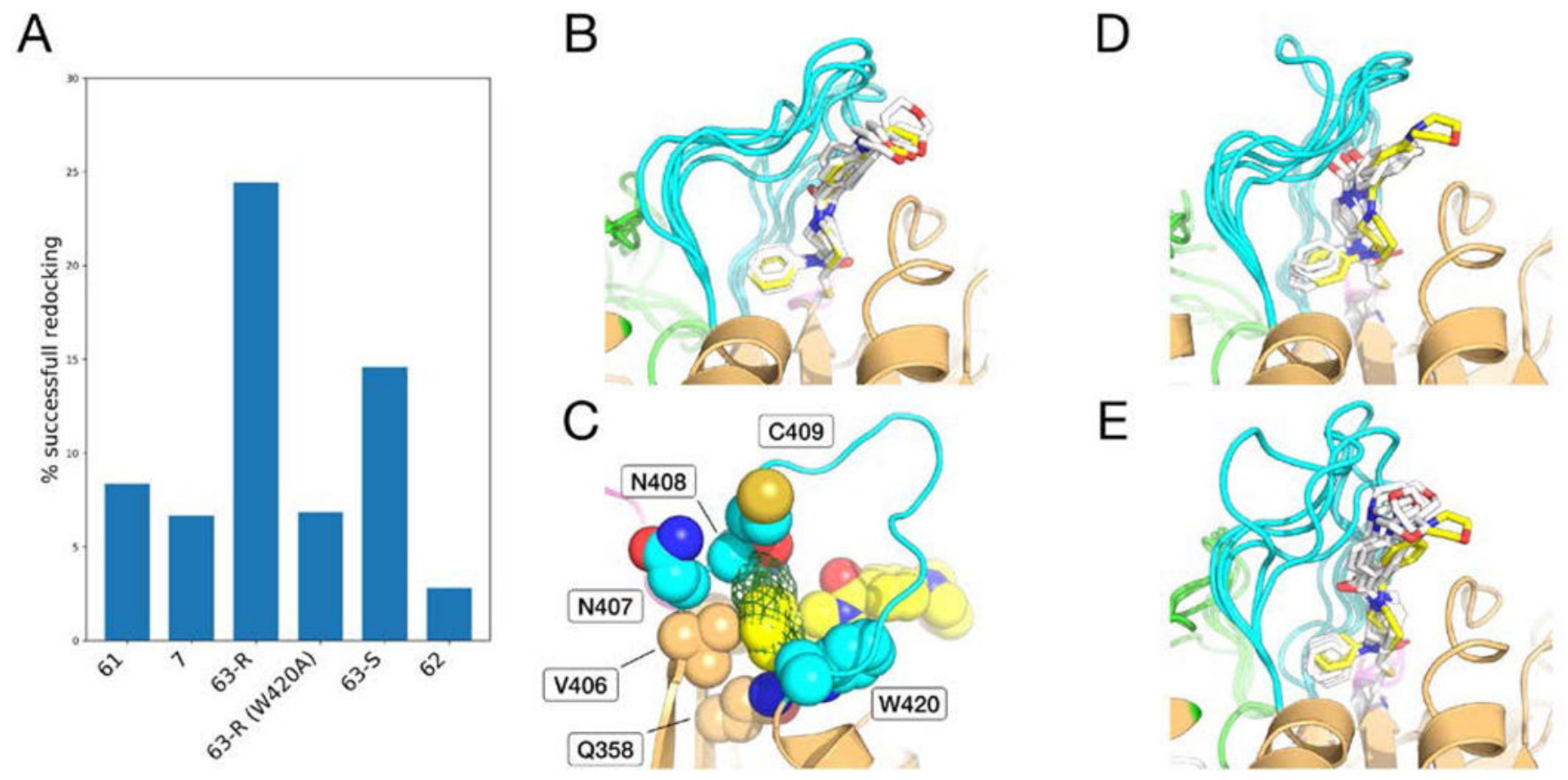

Figure 6.

Docking results analysis. (A) Cumulative docking success rates for 61, 7, 63-R, 63-S, and 62 on the 5000 loop models ensemble. (B) The top 5 docking results of $\mathbf{6 3 - R}$ (white carbon) with respect to the experimental coordinates (yellow carbon), with modeled loop 2

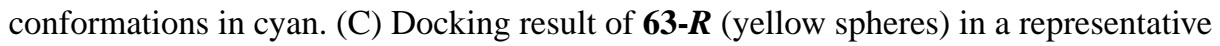
model, showing how the position of the naphthyl ring in the corresponding position (green mesh) would clash with the residues of the loop 2 and the folded portions of the protein (cyan and tan spheres, respectively). (D,E) The top 5 docking results of compounds 7 and 63-S. 

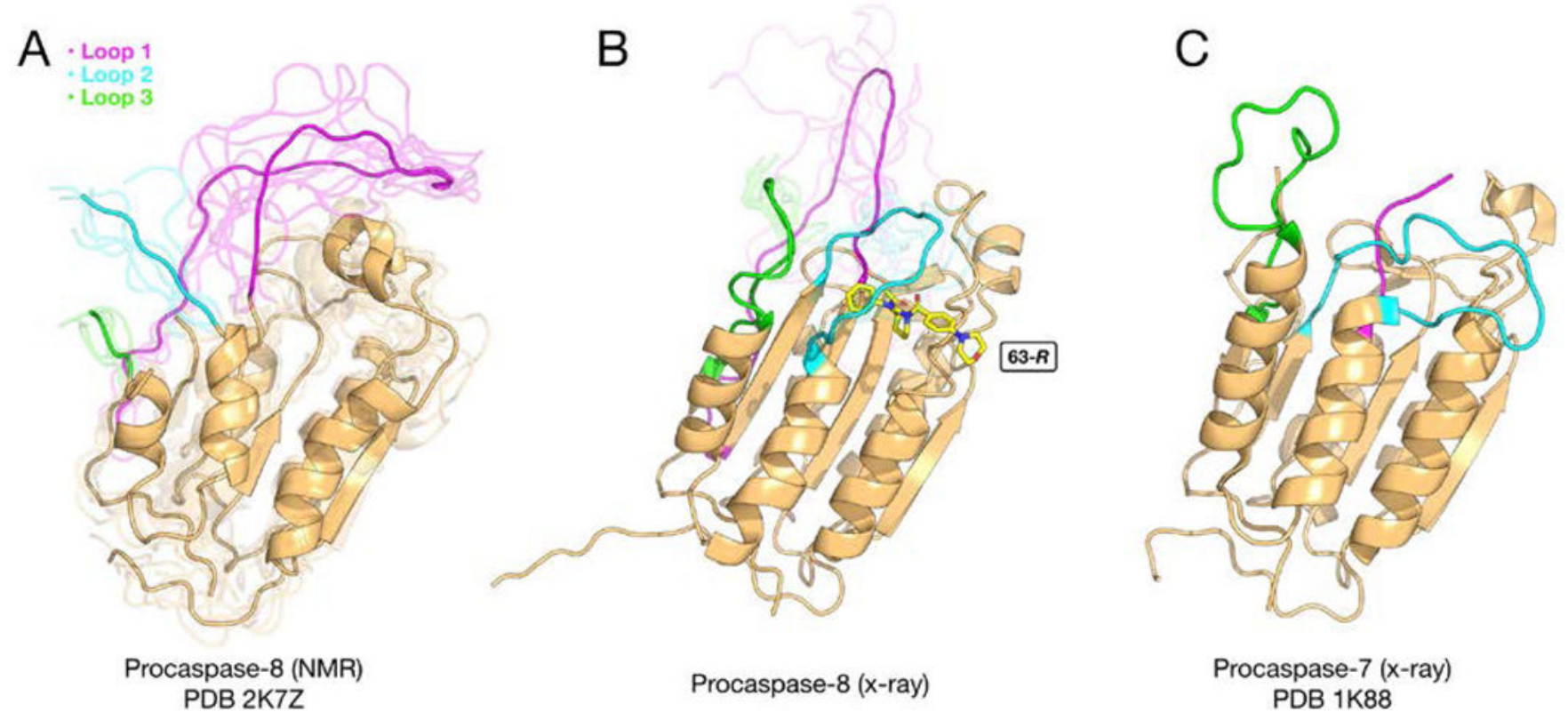

Figure 7.

Comparison of different procaspase protein structures. (A) Cartoon representation of the average NMR structure of procaspase- 8 (PDB 2k7z), obtained from 20 models, with the first five NMR models represented in transparent. The loops 1, 2, and 3 are colored magenta, cyan and green, respectively. (B) Average structure of procaspase-8 in complex with the inhibitor 63-R (chain B only) in stick representation and colored in yellow, obtained from the 1000 best loop modelling models, with the five best models represented in transparent. (C) X-ray structure of procaspase-7 (PDB 1k88) (chain B only) in cartoon representation with loops 1,2, and 3 colored magenta, cyan and green, respectively. 